\section{Image-Guided Cancer Therapy: A Multidisciplinary Approach}

\author{
D.E. Dupuy, Y. Fong, and W.N. McMullen, Eds.
}

New York, NY: Springer, 2013, 947 pages, $\$ 269$

Image-guided tumor ablation is a minimally invasive strategy to treat focal tumors by inducing irreversible cellular injury through the applications of thermal and, more recently, nonthermal energy or chemical injection. This approach has become a widely accepted technique and is incorporated into the treatment of a range of clinical circumstances, including tumors in the liver, lung, kidney, bone, and adrenal glands. Given the multiplicity of treatment types and potential complexity of paradigms in oncology, and the wider application of thermal ablation, a thorough understanding of the basic principles (goals and mechanisms) as well as recent advances in technology is a necessary prerequisite for their effective clinical use. The development of radiofrequency ablation serves as an excellent model for the evolution and development of additional ablation modalities. Besides technologic advancements in ablation devices, thermal ablation has been successfully combined with adjuvant chemotherapy and radiation.

Interventional oncology is a rapidly growing field that is already indispensable in the care of cancer patients. Needle- and catheter-based therapies provide palliation of symptoms, cytoreduction in cases of incurable cancers, and potential cure in cancers discovered at an early stage. This book is an attempt to summarize the updated physical and biologic basis of practice and current clinical delivery.

This comprehensive textbook, which was produced by 3 editors, 3 associate editors, 7 section editors, and 125 contributors who are outstanding leaders in the field, thoroughly demonstrates the current practice of interventional oncology. The authorship reflects the multidisciplinary nature of the field of cancer care by surgeons, medical and radiation oncologists, and interventional radiologists. The book is organized into both organ-based and specific topics. The 63 chapters allow the reader to become educated on all aspects of interventional oncology. The first part of the book describes the theories and science behind several techniques, including the newest, electroporation. The second part deals with the development of clinical practice, interactions with anesthesia, and management issues in cancer patients who are undergoing these procedures. The total management of the patient is not well discussed and is one of the most difficult aspects of interventional oncology practice. The later parts discuss therapies that are simply not covered in other resources. The information on prostate, breast, gynecologic, and pediatric cases offers an excellent complement to the basic material presented earlier in the book. Keen areas of interest such as hepatic, bone, and metastatic tumors are specifically updated and categorized.

The book is both readable and practical, with superb figures and useful tables. The references are updated, and the index is helpful. This timely, important, and outstanding contribution to the literature must be read by all physicians who are learning and practicing interventional oncology, especially cancer practitioners today.

\author{
E. Edmund Kim \\ University of California at Irvine \\ 101 The City Dr. S. \\ Orange, $C A 92868$ \\ E-mail: edmundek@uci.edu
}

